\title{
Dynamics of Discrete Time Systems with a Hysteresis Stop Operator
}

\author{
Maxim Arnold* Nikita Begun ${ }^{\dagger} \quad$ Pavel Gurevich ${ }^{\ddagger}$ Eyram Kwame* \\ Harbir Lamba ${ }^{\S} \quad$ Dmitrii Rachinskii*
}

\begin{abstract}
We consider a piecewise linear two-dimensional dynamical system that couples a linear equation with the so-called stop operator. Global dynamics and bifurcations of this system are studied depending on two parameters. The system is motivated by modifications to general-equilibrium macroeconomic models that attempt to capture the frictions and memory-dependence of realistic economic agents.
\end{abstract}

\section{Introduction}

The stop operator was proposed by L. Prandtl as an elementary model of quasistatic elastoplasticity [42], see Fig. 1.1(a). It presents a simple example of a rate-independent operator with local memory [47], and, as such, is used as an elementary building block for important models of hysteresis phenomena such as the Prandtl-Ishlinskii operator [31, the Preisach operator [30], and their generalizations [39]. Applications of these nonsmooth operators include modeling friction [44], elastoplastic materials [34], magnetic hysteresis [39], fatigue and damage counting [20,45], constitutive laws of smart materials [17, 25, 26], sorption hysteresis [1,9,33,41], and phase transitions [11. More recent applications range from biology and medicine [21, 24] to economics and finance [13, 15, 32]. On the other hand, stop can also be viewed as a solution operator of a simple variational inequality describing the Moreau sweeping process with rigid characteristic in one dimension [40], see Fig. 1.1(b).

Modeling of closed systems that exhibit hysteresis typically leads to differential equations which include the above nonsmooth operators. Dynamics of these systems have been analyzed with various techniques including topological degree methods [2,4,10,29], differential inclusions [35], switched systems [3], and energy considerations using the dissipative property of hysteresis [27]. As most of these models are motivated by engineering and physics applications, they are naturally formulated in continuous time setting. Discrete time systems with hysteresis operators have received little attention and were studied mostly in the context of numerical discretizations of continuous systems. However, the

\footnotetext{
*University of Texas at Dallas, TX, USA

$\dagger$ Free University of Berlin, Germany and Saint Petersburg State University, Russia

${ }_{\ddagger}^{\ddagger}$ Free University of Berlin, Germany and Peoples’ Frienship University of Russia, Russia

${ }^{\S}$ George Mason University, VA, USA
} 


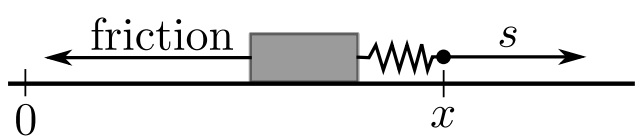

(a)

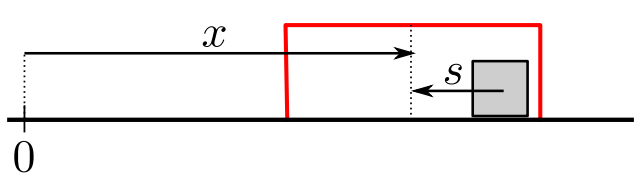

(b)

Figure 1.1: Interpretations of the stop operator. (a): Schematic of the Prandtl's model of quasi-static elastoplasticity: the box is not moving unless the absolute value of the force $s$ of the ideal spring reaches the maximal value $\rho$ of friction. (b): Schematic of the Moreau sweeping process with rigid characteristic in one dimension. The position $x$ of the center of the outer frame is the input; the relative position $s$ of the center of the frame with respect to the center of the box is the output. The frame moves and drags the box.

discrete time modeling is typical for certain applications, e.g., in economics, and one can expect that discrete time models motivated by such applications can exhibit interesting dynamical scenarios when nonsmooth hysteresis terms are included.

In this paper, we consider an example of a simple discrete time system which consists of a linear scalar equation coupled with the one-dimensional stop operator. This system can be equivalently written as a two-dimensional piecewise linear map. It has multiple equilibrium points which form a segment in the phase space. We present analysis of global dynamics and bifurcations depending on two parameters. In particular, the global attractor can consist of two semi-stable equilibrium points, a segment of stable equilibrium points, a segment of unstable equilibrium points, a 2-periodic orbit, a 2-periodic orbit and two semi-stable equilibrium points, and, in a critical case, a two-dimensional set of equilibrium and 2-periodic points. For a certain open set of parameter values, the system possesses infinitely many unstable periodic orbits.

The paper is organized as follows. In the next section, we present the main results. In Section 3, a motivating economics example is discussed. In a standard setting of a general-equilibrium macroeconomic model, we propose modeling stickiness in agent's expectations by the play operator dual to the stop. This leads us to a four-dimensional system containing the stop operator, and we present a few numerical examples of its dynamics. The two-dimensional system discussed in Section 2 can be considered as a simple prototype counterpart of this higher dimensional economic model. The last section contains the proofs.

\section{Main results}

Let $s_{0} \in[-1,1]$ and let $\left\{x_{n}\right\}, n \in \mathbb{N}_{0}$, be a real-valued sequence. The stop operator $S$ maps a pair $s_{0},\left\{x_{n}\right\}$ to a sequence defined by the formula

$$
s_{n+1}=\Phi\left(s_{n}+x_{n+1}-x_{n}\right), \quad n \in \mathbb{N}_{0},
$$

where

$$
\Phi(\tau)=\left\{\begin{array}{rll}
-1 & \text { if } & \tau<-1 \\
\tau & \text { if } & |\tau| \leq 1 \\
1 & \text { if } & \tau>1
\end{array}\right.
$$


see Fig. 2.1(a), Here $s_{0}$ is called the initial state, $\left\{x_{n}\right\}$ is called the input, and $\left\{s_{n}\right\}$ is called the output (or, the variable state) of the stop operator. The operator that maps the pair $s_{0},\left\{x_{n}\right\}$ to the sequence $\left\{x_{n}-s_{n}\right\}$ is called the play operator (see Fig. 2.1(b)).

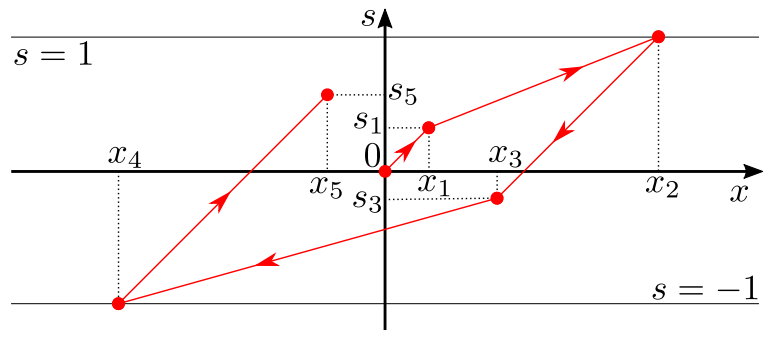

(a)

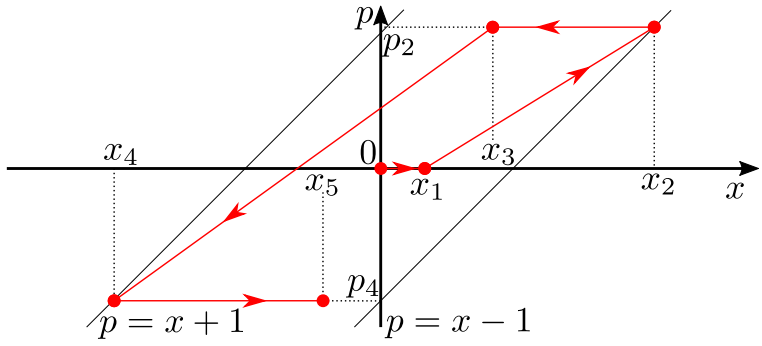

(b)

Figure 2.1: Interpretations of the stop and play operators. (a): An example of the input-output sequence of the stop operator: $(0,0)=\left(x_{0}, s_{0}\right),\left(x_{1}, s_{1}\right),\left(x_{2}, 1\right),\left(x_{3}, s_{3}\right)$, $\left(x_{4},-1\right),\left(x_{5}, s_{5}\right)$. (b): An example of the input-output sequence of the play operator: $(0,0)=\left(x_{0}, p_{0}\right),\left(x_{1}, p_{0}\right),\left(x_{2}, p_{2}\right),\left(x_{3}, p_{2}\right),\left(x_{4}, p_{4}\right),\left(x_{5}, p_{4}\right)$.

Coupling the output of the stop operator with the input sequence via a linear transformation with real-valued coefficients $\lambda$ and $a$, we consider the dynamical system

$$
\left\{\begin{array}{l}
x_{n+1}=\lambda x_{n}+a s_{n}, \\
s_{n+1}=\Phi\left(s_{n}+x_{n+1}-x_{n}\right)
\end{array}\right.
$$

with $n \in \mathbb{N}_{0}$ on the strip $L=\{(x, s): x \in \mathbb{R}, s \in[-1,1]\}$. From hereon we assume that $|\lambda|<1$. This inequality ensures that all the trajectories of system (2.2) are bounded.

It is easy to see that the equilibrium points of system 2.2 form the segment

$$
E F=\left\{(x, s): x=\frac{a s}{1-\lambda},-1 \leq s \leq 1\right\}
$$

with the end points

$$
E=\left(x^{*}, 1\right)=\left(\frac{a}{1-\lambda}, 1\right), \quad F=\left(-x^{*},-1\right)=\left(-\frac{a}{1-\lambda},-1\right) .
$$

We use the standard notion of stability and instability (in the Lyapunov sense) for equilibria and periodic orbits. We will also say that an equilibrium point $\left(x_{e}, s_{e}\right)$ of system (2.2) is semi-stable if there are open sets $U_{1}, U_{2} \subset\{(x, s):|s|<1\}$ such that $\left(x_{e}, s_{e}\right)$ belongs to their boundaries and simultaneously:

- for every $\varepsilon>0$ there is a $\delta>0$ such that any trajectory starting from the $\delta$-neighborhood of the equilibrium point $\left(x_{e}, s_{e}\right)$ in the set $U_{1}$ belongs to the $\varepsilon$ neighborhood of $\left(x_{e}, s_{e}\right)$ for all positive $n$;

- there is an $\varepsilon_{0}>0$ such that any trajectory starting in $U_{2}$ leaves the $\varepsilon_{0}$-neighborhood of the equilibrium $\left(x_{e}, s_{e}\right)$ after a finite number of iterations. 


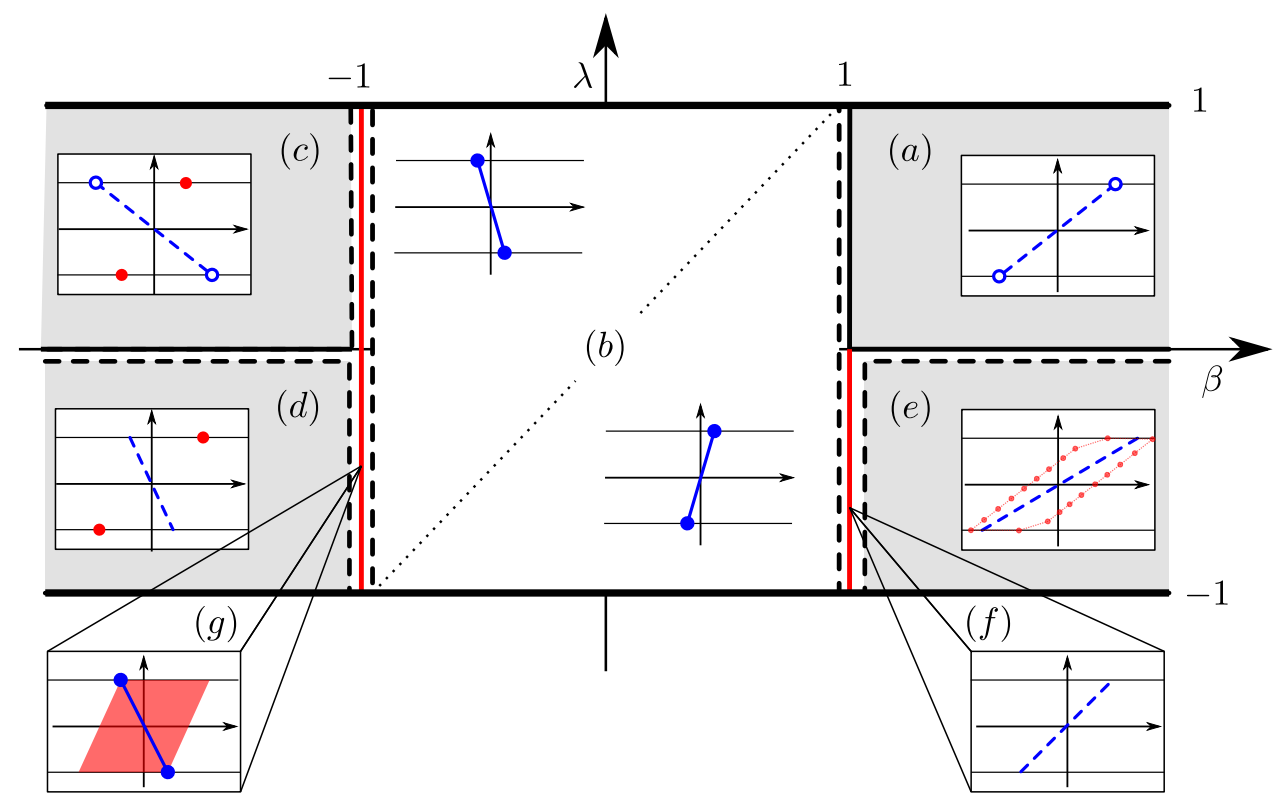

Figure 2.2: Bifurcation diagram. The segment $E F$ of the fixed points is shown by the blue line. Stable fixed points are denoted by the solid line, unstable fixed points are shown by the dashed line. Stable end-points of EF are shown as filled blue discs; semistable points are denoted by empty blue discs; in the unstable case, no special notation is used. The dotted line in case $(b)$ corresponds to the set of parameters leading to the infinite slope of the line EF. Periodic points are shown in red. Filled red discs in cases $(c)$ and $(d)$ correspond to the stable 2-periodic orbit $\pm Q$; the red parallelogram in case $(g)$ consists of stable 2-periodic orbits. Case (e) corresponds to complex dynamics when the system has periodic orbits with arbitrary large periods (see Theorem 2). One such orbit is sketched on the diagram. In the critical case $(f)$, the segment $E F$ attracts all the trajectories.

Our main result consists in the classification of the long time behavior for the orbits of system (2.2). Dynamics of system (2.2) depends on the values of the parameters $\lambda$ and $\beta=\lambda+a$ as described in the Theorem 1 (see also the diagram in Fig. 2.2).

Theorem 1. Let $\beta=\lambda+a$ and $|\lambda|<1$.

(a) If $\lambda \geq 0, \beta \geq 1$, then the equilibrium points $E$ and $F$ are semi-stable and all the other equilibrium points are unstable. Each non-equilibrium trajectory either converges to $E$ or to $F$.

(b) If $|\beta|<1$, then all the equilibrium points are stable and each trajectory of system (2.2) converges to an equilibrium point.

(c) If $\lambda \geq 0, \beta<-1$, then the points $E$ and $F$ are semi-stable, all the other equilibrium points are unstable, and there exists a stable 2-periodic orbit

$$
\pm Q=\left(\mp \frac{a}{1+\lambda}, \pm 1\right)
$$

Each non-equilibrium trajectory either converges to $E$ or to $F$ or to the orbit (2.4). 
(d) If $\lambda<0, \beta<-1$, then all the equilibrium points are unstable. Each non-equilibrium trajectory converges to the stable 2-periodic orbit (2.4).

(e) If $\lambda<0, \beta>1$, then all the equilibrium points are unstable. System (2.2) has periodic orbits of all sufficiently large periods. At most one periodic orbit is stable.

(f) If $\lambda<0, \beta=1$, then all the equilibrium points are unstable. Each trajectory either ends up at $E$ or at $F$, or converges to the segment $E F$.

(g) If $\beta=-1$, then all the equilibrium points are stable. The parallelogram

$$
\Sigma=\left\{(x, s): 2 \frac{(1-\lambda) x-a}{1-\lambda+a}+1 \leq s \leq 2 \frac{(1-\lambda)(x-1)}{1-\lambda+a}+1,|s| \leq 1\right\}
$$

with the vertices $E, F, Q=(1,1)$ and $-Q=(-1,-1)$ consists of stable 2-periodic orbits and the diagonal EF of fixed points. Every non-equilibrium trajectory converges either to one of the equilibrium points $E$ or $F$, or to a 2-periodic orbit in the parallelogram $\Sigma$.

The existence of infinitely many periodic orbits in case $(e)$ may indicate the presence of a global strange attractor or a chaotic attractor co-existing with the stable periodic orbit. More detailed analysis of this case will be a subject of future work.

Theorem 1 describes several bifurcation scenarios. In particular, the period doubling scenario is interesting because the stable 2-periodic orbit, which exists for $\beta<-1$ (see cases $(c)$ and $(d)$ is not close to any equilibrium point (as would be typical for smooth systems). Let us consider $\beta$ as a decreasing bifurcation parameter. When this parameter crosses the value -1 , the equilibrium points of the segment $E F$, which are stable for $\beta \in(-1,1)$ (see case $(b)$ ), destabilize and the 2-periodic orbit (2.4) appears away from the segment $E F$. This transition is accompanied by the creation of the parallelogram $\Sigma$ filled with 2 -periodic orbits at the critical value $\beta=-1$. This parallelogram is spanned by the 2-periodic orbit $\pm Q=( \pm 1, \pm 1)$ and the equilibrium points $E, F$ (case $(g)$ ).

Assume that $\lambda<0$. When the parameter $\beta$ increases and crosses the value 1 , the equilibrium points destabilize and infinitely many periodic orbits appear (see case $(e)$ ). Dynamics for the critical value $\beta=1$ is described by case $(f)$. The following theorem complements case $(e)$ of Theorem 1 .

Theorem 2. Assume that the conditions of case (e) of Theorem 1 hold and hence system (2.2) has infinitely many periodic orbits, of which at most one is stable. Then the relation $(\lambda, \beta) \in \Omega_{k}$ with

$$
\Omega_{k}=\left\{(\lambda, \beta): \frac{\beta^{k}-1}{\beta-1} \leq-\frac{1}{\lambda}<\beta^{k}, \beta>1,-\frac{1}{\lambda}>1\right\},
$$

where $k \in \mathbb{N}$, ensures that system $(2.2)$ has a unique stable $(2 k+2)$-periodic orbit. If $(\lambda, \beta) \notin \bigcup_{k=1}^{\infty} \Omega_{k}$, then all the periodic orbits are unstable.

Remark 1. The domains $\Omega_{k}$ of existence of stable periodic orbits with different periods do not intersect (see Fig. 2.3).

Remark 2. It will follow from the proof of Theorem 2 that if $(\lambda, \beta)$ belongs to the interior of $\Omega_{k}$ for some $k$, then the corresponding stable periodic orbit is asymptotically stable. 


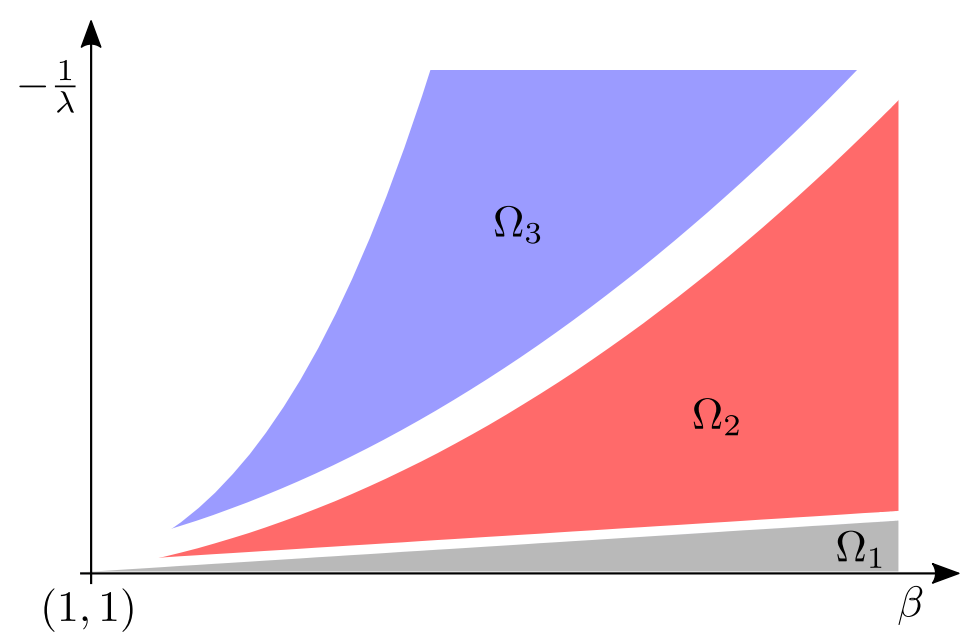

Figure 2.3: Domains $\Omega_{k}$ of existence of a (unique) stable periodic orbit of period $2 k+2$ in the coordinates $\beta=\lambda+a>1$ and $-1 / \lambda>1$ for case $(e)$ of Theorem 1 .

\section{Discussion}

Hysteresis effects, which are well known in engineering and physics, have become a topic of interest in other disciplines. In economics, hysteresis has been well documented in the relationship between the output of the economy and unemployment rate [5]. Hysteresis has been also closely associated with other stylized facts such as path dependence [8, 22], stickiness of prices and information [6, 19,38], and heterostasis (multiplicity of equilibria) [14] that describe empirical economic data. An attempt to obtain quantitative models of these empirical observations naturally motivated the use of the play operator and more complex models of hysteresis developed in physics in the economic context. For example, the play operator was shown to produce a good model of the dependence of supply and demand on the price [7, 23]. This model was fitted to microeconomic data based on a survey of German beer exports. It replaces the demand and supply curves by play operators and predicts well the observed price rigidity. The Preisach operator has been applied to modeling hysteresis in unemployment [16]. Furthermore, the phenomenology of these hysteresis models is compatible with the multi-agent modeling framework typical for economic models [13, 36, 37].

The next natural step towards modeling the above effects in economics would consist in formulation and analysis of closed models. With this motivation, let us consider the following system, which belongs to the class of the so-called Dynamic Stochastic General Equilibrium Models (DSGE) of macroeconomics (see, e.g., 12, 28, 43, 46]):

$$
\left\{\begin{array}{l}
y_{n+1}=y_{n}-a_{1}\left(v_{n+1}-\sigma_{n+1}\right)+\varepsilon_{n} \\
u_{n+1}=b_{1} \sigma_{n+1}+\left(1-b_{1}\right) u_{n}+b_{2} y_{n+1}+\eta_{n} \\
v_{n+1}=c_{1}\left(u_{n+1}-u^{*}\right)+c_{2} y_{n+1}+c_{3} v_{n}+\xi_{n}
\end{array}\right.
$$

where $y_{n}$ is the output gap (or employment rate, or another measure of activity of the economy), $u_{n}$ is the rate of inflation, $v_{n}$ is the interest rate, $\sigma_{n}$ is the aggregate of the economic agents' expectation of the future inflation rate, and $\varepsilon_{n}, \eta_{n}, \xi_{n}$ are exogenous 


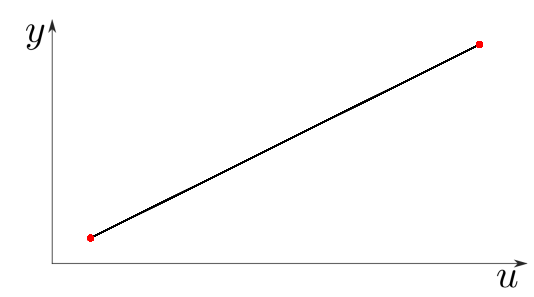

(a)

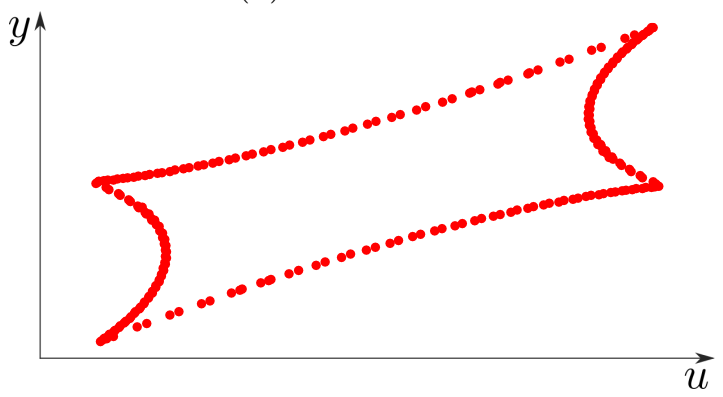

(d)

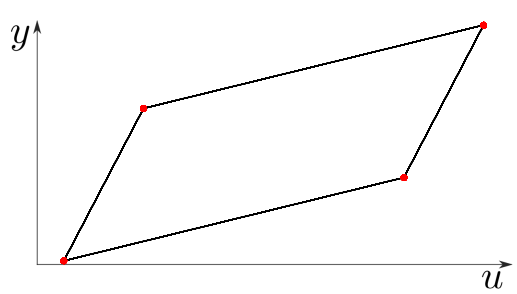

(b)

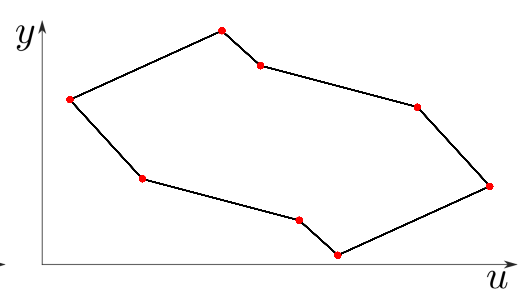

(c)

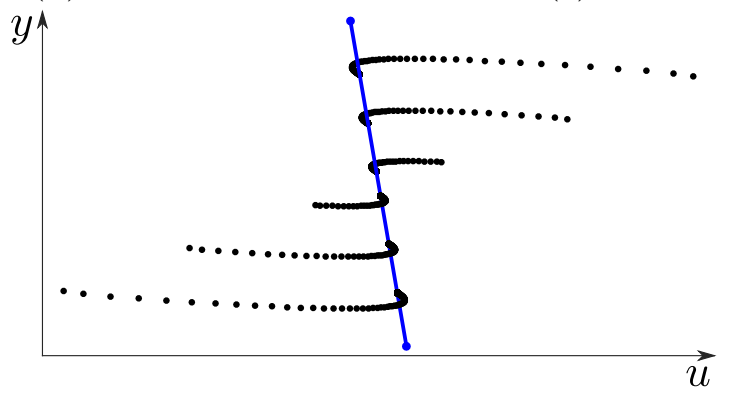

(e)

Figure 3.1: Projections of various trajectories of system (3.1)-3.2) onto $(u, y)$ plane. Parameter $\rho$ is fixed to be 1 , all the other parameters are given in the format $a_{1}, b=\left(b_{1}, b_{2}\right)$, $c=\left(c_{1}, c_{2}, c_{3}\right)$. (a): 2-periodic orbit for $a_{1}=0.99, b=(0.76,0.9), c=(1.4,9.7,0.025)$. (b): 4-periodic orbit for $a_{1}=0.7, b=(0.75,0.5), c=(4.8,3.6,3.45)$. (c): 8-periodic orbit for $a_{1}=0.9, b=(0.73,0.9), c=(1.2,3.15,1.3)$. (d): Quasi-periodic orbit for $a_{1}=0.7$, $b=(0.7,0.55), c=(4.8,4.15,3.8)$. (e): Trajectories converging to different points of the segment of equilibrium points for $a_{1}=0.01, b=(0.01,0.03), c=(1,6,0.54)$.

noise terms, see [18]. All the parameters are non-negative, $b_{1}<1$, and the parameter $u^{*}$, the inflation target, is for convenience set to zero.

In order to close the model, we need to complement system (3.1) with an equation defining how the economic agents' expectation of the future inflation rate $\sigma_{n}$ is related to the actual inflation rate $u_{n}$. We assume that $\sigma_{n}$ is related to $u_{n}$ via the play operator:

$$
\sigma_{n+1}=u_{n+1}-\rho s_{n+1}, \quad s_{n+1}=\Phi\left(s_{n}+\rho^{-1}\left(u_{n+1}-u_{n}\right)\right),
$$

where $\Phi$ is function (2.1), see Fig. 2.1(b), Note that the sequence $s_{n}$ is the output of the stop operator with the input $\rho^{-1} u_{n}$, see Fig. 2.1(a), The play operator introduces inertia, or stickiness (with the associated memory), into the response of aggregated agent's expectation of inflation to variations of the rate of inflation $u_{n}$. The parameter $\rho>0$ measures the maximum deviation of the expected rate of inflation from the actual rate.

Let us consider the unperturbed system (3.1), (3.2), i.e., we set the noise terms $\varepsilon_{n}$, $\eta_{n}, \xi_{n}$ to zero. This autonomous system can be rewritten in the explicit form

$$
z_{n+1}=\Lambda z_{n}+A s_{n}, \quad s_{n+1}=\Phi\left(s_{n}+\rho^{-1}\left(u_{n+1}-u_{n}\right)\right),
$$

where $z$ is the column vector $z=(y, u, v)^{T}, \Lambda$ is a $3 \times 3$ matrix, and $A \in \mathbb{R}^{3}$. Therefore, system 2.2 can be viewed as a simpler one-dimensional counterpart of system (3.1), (3.2).

Fig. 3.1 presents various attractors of model (3.1), (3.2) obtained numerically for different parameter regimes. In particular, trajectories can converge to stable equilibrium 
points that form a segment in the phase space, see Fig. 3.1(e). Alternatively, one can observe convergence to a 2-periodic orbit, or to a periodic orbit of a higher period, which coexists with the set of unstable equilibrium points (see Figs. 3.1(a) 3.1(c)]. Fig. 3.1(d) indicates a possibility of quasiperiodic dynamics. Comparing these scenarios with different cases of Theorem 1 suggests that the prototype model 2.2 can help understand some features of dynamics of the more complex macroeconomic model (3.1), (3.2). Analysis of the latter model is beyond the scope of this paper and will be the subject of future work.

\section{Proofs}

We will prove statements of Theorem 1 in the counter-clockwise order along the bifurcation diagram in Fig. 2.2. Thus, we prove case $(a)$ in Section 4.1 , then case $(b)$ for non-negative $\lambda$ in Section 4.2. Proofs for cases $(c)$ and $(d)$ are presented in Sections 4.3 and Section 4.4, respectively. In Section 4.5, we present the proof of the remaining part of case $(b)$ for negative $\lambda$. Proofs of case $(e)$ and of Theorem 2 are presented in Section 4.6 . Finally, Sections 4.7 and 4.8 contain the proofs for critical cases $(f)$ and $(g)$, respectively.

We use the following notations: $A_{x}$ and $A_{s}$ will denote the $x$ and $s$ coordinates of a point $A$ in the $(x, s)$-plain. Transformation $(2.2)$ will be denoted by $f$. Throughout the proofs, we will use the variable $p=x-s$ (output of the play operator, see Fig. 2.1(b)). We will denote by $A_{p}=A_{x}-A_{s}$ the $p$-coordinate of a point $A$.

Let us start with a few preliminary remarks. First, due to the fact that

$$
f(-x,-s)=-f(x, s)
$$

it is sufficient to present the proofs for a half of the phase space.

Lemma 1. For any point $A$ to the left of the segment $E F$, one has $[f(A)]_{x}>A_{x}$. For any point $B$ to the right of the segment $E F$, one has $[f(B)]_{x}<B_{x}$.

Proof. Since $A$ lies to the left of the segment $E F$, one has $(1-\lambda) A_{x}<a A_{s}$. Thus $[f(A)]_{x}=\lambda A_{x}+a A_{s}>\lambda A_{x}+(1-\lambda) A_{x}=A_{x}$. The second statement follows from (4.1).

Lemma 2. Let $\beta>0$. Then for any two points $A$ and $B$ with the same $p$-coordinate $A_{p}=B_{p}$, from $A_{x}>B_{x}$ it follows $[f(A)]_{x}>[f(B)]_{x}$.

Proof. It suffices to note that $[f(A)]_{x}=\lambda A_{x}+a A_{s}=\beta A_{x}-a A_{p}$.

Denote by $\Pi \subset L$ the parallelogram with the diagonal $E F$, two sides on the lines $s= \pm 1$, and two sides with slope 1 :

$$
\Pi=\left\{(x, s):|x-s| \leq\left|\frac{a}{1-\lambda}-1\right|,|s| \leq 1\right\} .
$$

Lemma 3. For $0 \leq \lambda<1, \beta \leq 0$ and for $-1<\lambda \leq 0,-1 \leq \beta \leq 0$ the parallelogram $\Pi$ is invariant under the map (2.2). 
Proof. Let $\left(x_{n}, s_{n}\right) \in \Pi$, i.e. $\left|p_{n}\right| \leq \frac{1-\beta}{1-\lambda}$. Since the upper-right and the lower-left vertices of $\Pi$ are the points $E^{\prime}=\left(-\frac{a}{1-\lambda}+2,1\right)$ and $F^{\prime}=\left(\frac{a}{1-\lambda}-2,-1\right)$, respectively, it suffices to prove that

$$
\left|x_{n+1}\right| \leq-\frac{a}{1-\lambda}+2
$$

If $0 \leq \lambda<1, \beta \leq 0$, then

$$
\left|x_{n+1}\right| \leq \lambda \frac{1-\beta}{1-\lambda}-\beta=-\frac{a}{1-\lambda},
$$

which implies (4.3). If $-1<\lambda \leq 0,-1 \leq \beta \leq 0$, then

$$
\left|x_{n+1}\right| \leq-\lambda \frac{1-\beta}{1-\lambda}-\beta,
$$

which yields 4.3 because $\lambda<1$ and $\lambda \beta \leq 1$.

\subsection{Case $(a)$}

In this case, $\lambda \geq 0$ and $\beta \geq 1$. Therefore $a>0$ and the slope of the segment $E F$ of equilibrium points is positive and less than or equal to 1 as shown in Fig. 4.1.

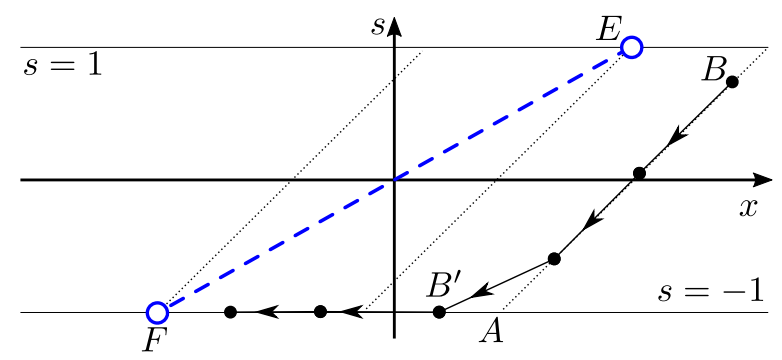

Figure 4.1: $\lambda \geq 0, \beta \geq 1$. A trajectory starting at a point $B$ to the right of the segment $E F$ converges to the fixed point $F$. Dotted lines have slope 1 .

Consider a point $B$ which lies to the right of the segment $E F$. Denote by $A$ the intersection point of the lines $p=B_{p}$ and $s=-1$. Let $B^{\prime}$ denote the point at which the trajectory $\left\{f^{n}(B)\right\}$ hits the line $s=-1$ for the first time. From Lemma 1 it follows that $\left[f^{-1}\left(B^{\prime}\right)\right]_{x}>A_{x}$, and $[f(A)]_{x}>F_{x}$ since $\lambda>0$. Thus, from Lemma 2 we obtain $B_{x}^{\prime}>F_{x}$. Since $B_{s}^{\prime}=-1$, it follows that $\left[f\left(B^{\prime}\right)\right]_{x}-F_{x}=\lambda\left(B_{x}^{\prime}-F_{x}\right)$. Hence, due to $\lambda \in[0,1)$, the trajectory converges to the equilibrium $F$ along the line $s=-1$ (see Fig. 4.1). We conclude that every trajectory that starts to the right of the segment $E F$ of equilibrium points, converges to $F$. Every trajectory which starts to the left of $E F$ converges to $E$ due to 4.1 .

\subsection{Case $(b), \lambda \geq 0$}

\subsection{1 $0<\beta<1$}

In this case, the segment $E F$ has a positive slope greater than 1 if $a>0$ and nonpositive if $a \leq 0$. 
1 First, let us consider the trajectory of a point $A$ that belongs to the parallelogram $\Pi$ defined by 4.2 , see Fig. 4.2 . Denote by $P^{*}$ the point of intersection of the line $p=A_{p}$ with the segment $E F$ of equilibrium points. Since

$$
[f(A)]_{p}=A_{p}, \quad[f(A)]_{x}-P_{x}^{*}=\beta\left(A_{x}-P_{x}^{*}\right)
$$

with $\beta \in(0,1)$, the trajectory of $A$ converges to the point $P^{*}$.

2 Thanks to Lemma 2, all the other trajectories that start to the right of the parallelogram $\Pi$, move down along the line $p=$ const until they hit the line $s=-1$ and then monotonically converge to the equilibrium point $F$ along this line from the right, see Fig. $4.2(\mathrm{a})$.

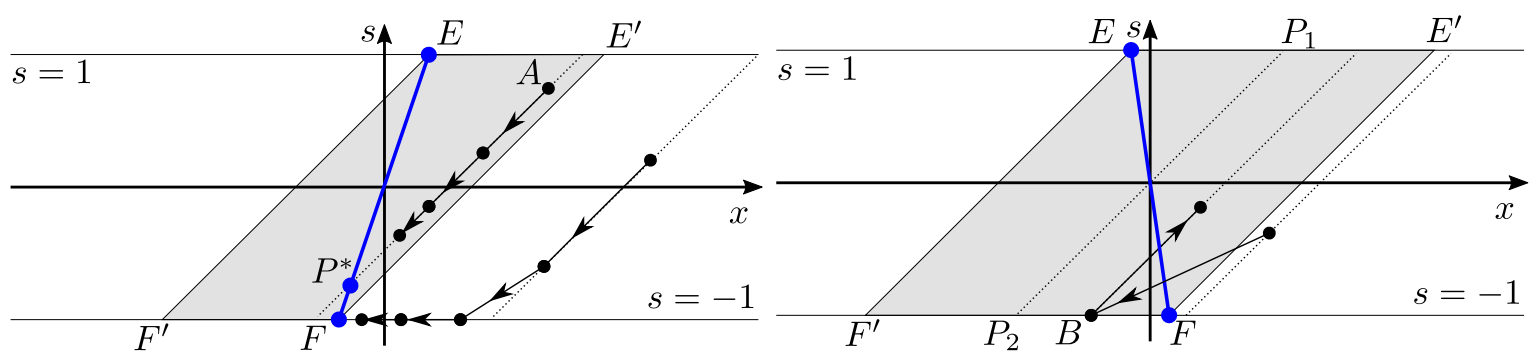

(a) $0<\beta<1$.

(b) $-1<\beta \leq 0$.

Figure 4.2: Theorem $1(b)$, Case $\lambda \geq 0$. Dotted lines have slope 1 . The shaded area is the parallelogram $\Pi=E E^{\prime} F F^{\prime}$.

\subsection{2 $-1<\beta \leq 0$}

In this case, $a<0$ and the segment $E F$ has a negative slope as in Fig. 4.2(b),

1 If a trajectory starts to the right of the parallelogram $\Pi$, then, since $\beta \leq 0$, it hits the line $s=-1$ after one iteration. If it hits the line to the right of the equilibrium $F$, then the trajectory converges to this equilibrium along the line $s=-1$ from the right due to $\lambda \geq 0$. On the other hand, if this trajectory hits the line $s=-1$ at a point $B$ to the left of the point $F$, then $B$ belongs to the parallelogram $\Pi$. In order to show this, we note that for the previous point $f^{-1}(B)=\left(x_{n}, s_{n}\right)$, we have

$$
x_{n}-s_{n}>-\frac{a}{1-\lambda}+1,
$$

because the point $\left(x_{n}, s_{n}\right)$ lies to the right of the parallelogram $\Pi$. Therefore,

$$
\begin{aligned}
x_{n+1} & =\lambda x_{n}+a s_{n}>\lambda\left(-\frac{a}{1-\lambda}+1+s_{n}\right)+a s_{n} \\
& \geq \lambda\left(-\frac{a}{1-\lambda}+1\right)+\lambda+a .
\end{aligned}
$$

This last expression is greater than $F_{x}^{\prime}=\frac{a}{1-\lambda}-2$, which is the $x$-coordinate of the lower left vertex of the parallelogram $\Pi$. 
2 Consider points on the horizontal sides of $\Pi$. To be definite, assume that $s_{n}=$ -1. Denote by $P_{1}=(1,1)$ and $P_{2}=(-1,-1)$ the middle points of $E E^{\prime}$ and $F F^{\prime}$, respectively. If $\left(x_{n}, s_{n}\right) \in P_{2} F$, then the trajectory converges to the equilibrium along the line $p=$ const. Let $\left(x_{n}, s_{n}\right) \in F^{\prime} P_{2}$. If $s_{n+1}<1$, then the trajectory converges to the equilibrium along the line $p=$ const. If $s_{n+1}=1$, then $\left(x_{n+1}, s_{n+1}\right) \in E P_{1}$ since $x_{n+1}=\lambda x_{n}-a \leq-\lambda-a<1$. Again, the trajectory converges to the equilibrium along the line $p=$ const.

It remains to consider points in $\Pi$ that belong to the open band $|s|<1$. A trajectory starting from such a point either converges to an equilibrium along the line $p=$ const without hitting the lines $s= \pm 1$, or hits one of these lines and then converges to an equilibrium as discussed above.

\subsection{Case $(c)$}

As in the previous case, $a<0$ and the segment $E F$ has a negative slope (see Fig. 4.3). In this case, there is a 2-periodic orbit. A 2-periodic orbit consists of the points $\pm Q$ where $Q=\left(Q_{x}, 1\right)$. Here, $Q_{x}=-\lambda Q_{x}-a$, therefore

$$
Q_{x}=-\frac{a}{1+\lambda}
$$

Since $\beta<-1$, it follows that $Q_{x}>1$, so the distance between the $x$-components of $Q$ and $-Q$ is larger than 2 and thus periodic points indeed belong to the lines $s= \pm 1$.

1 Now we consider dynamics of different trajectories. Denote

$$
A=\left(\frac{a+2}{1-\lambda}, 1\right), \quad B=\left(\frac{1}{\lambda}\left(-\frac{a+2}{1-\lambda}-a\right), 1\right) .
$$

If $\lambda=0$, we formally set $B_{x}=\infty$ and replace the segment $A B$ below by the corresponding half-line. Note that $B_{x}>A_{x}$ and $Q \in A B$.

Lemma 4. Under the hypothesis of Theorem 1 (c), the segment $A B$ is invariant under the second iteration $f^{2}$ of the map $f$ and $f^{2}$ is a contraction on $A B$.

Proof. First, we note that if a point $\left(x_{n}, s_{n}\right)$ lies on the line $s=1$ to the right of the point $A$, then the image $\left(x_{n+1}, s_{n+1}\right)=f\left(x_{n}, s_{n}\right)$ of this point under the map $(2.2)$ belongs to the line $s=-1$. Indeed,

$$
x_{n+1}-x_{n}=\lambda x_{n}+a-x_{n} \leq(\lambda-1) A_{x}+a=-2,
$$

which implies $s_{n+1}=-1$. By (4.1), the points of the line $s=-1$ lying to the left of $-A$ are mapped to the line $s=1$. Furthermore, if a point $\left(x_{n}, s_{n}\right)$ lies on the line $s=1$ between the points $A$ and $B$, then its image $f\left(x_{n}, s_{n}\right)=\left(\lambda x_{n}+a, s_{n+1}\right)$ lies on the line $s=-1$ to the left of the point $-A$, and therefore the second iteration $f^{2}\left(x_{n}, s_{n}\right)$ belongs to the line $s=1$. Hence, the segment $A B$ is mapped by the second iteration $f^{2}$ to the line $s=1$. If $\left(x_{n}, 1\right) \in A B$, then $f^{2}\left(x_{n}, 1\right)=\left(\lambda^{2} x_{n}+\lambda a-a, 1\right)$. In particular, $\left[f^{2}(A)\right]_{x}$ is defined by the expression

$$
\lambda^{2} A_{x}+\lambda a-a=\frac{-a+2 \lambda^{2}+2 a \lambda}{1-\lambda} .
$$


The inequality

$$
\frac{-a+2 \lambda^{2}+2 a \lambda}{1-\lambda}>\frac{a+2}{1-\lambda},
$$

which is equivalent to $(\lambda-1)(\beta+1)>0$, ensures that $\left[f^{2}(A)\right]_{x} \geq A_{x}$.

To show that $\left[f^{2}(B)\right]_{x} \leq B_{x}$, it is sufficient to check the following inequality:

$$
\lambda^{2}\left(\frac{-2 a-2+a \lambda}{\lambda(1-\lambda)}\right)+a(\lambda-1)<\frac{-2 a-2+a \lambda}{\lambda(1-\lambda)} .
$$

After a simple manipulation, this inequality follows from $\beta+1<0$. Since $f^{2}$ maps $A$ and $B$ into $A B$ and $\left[f^{2}(x, 1)\right]_{x}$ is increasing on $A B$ with respect to $x$, we conclude that $f^{2}(A B) \subseteq A B$. Since $\lambda^{2}<1$, we see that $f^{2}$ is a contraction on $A B$, hence the trajectories starting in $A B$ converge to the fixed point $Q=\left(\frac{-a}{1+\lambda}, 1\right)$ of $f^{2}$, which belongs to the segment $A B$.

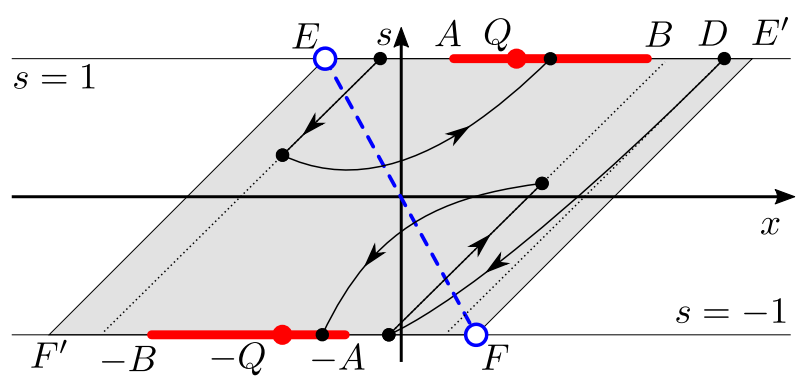

Figure 4.3: Case $\lambda \geq 0, \beta<-1$. Each of the red segments $A B$ and $-A B$ is mapped into itself by $f^{2}$.

2 Next, we consider the situations where $B$ lies to the right of $E^{\prime}$ and where $B$ lies between $A$ and $E^{\prime}$, respectively. In the former case, any trajectory starting between $A$ and $E^{\prime}$ converges to the 2-periodic orbit due to the above argument. Consider the latter case. Let a trajectory start on the upper side of the parallelogram $\Pi$ to the right of the point $B$ at a point $D=\left(x_{n}, 1\right)$, see Fig. 4.3. The image $f(D)=\left(x_{n+1},-1\right)$ of this point lie on the line $s=-1$ to the right of the point $-A$. Therefore, $f^{2}(D)$ belong to the interior of the strip $L$. Since $\beta<-1$, further iterations $f^{n+k}(D)$ belong to the line $s=-1$ for odd $k$ and to the interior of $L$ for even $k$, and the $x$-coordinate of the odd iterations monotonically decreases until the trajectory reaches the half-line $\left\{(x, s): x \leq-A_{x}, s=-1\right\}$. Without loss of generality, we can assume that $f(D)$ is the last point of the trajectory, which is still to the right of the point $-A$ on the line $s=-1$. Let us show that the point $\left(x_{n+3},-1\right)$ lies to the right of the point $\left(\frac{a}{1+\lambda},-1\right)$. To this end, we note that

$$
\begin{gathered}
\left(x_{n+2}, s_{n+2}\right)=\left(\lambda x_{n+1}-a,-1+\lambda x_{n+1}-a-x_{n+1}\right), \\
x_{n+3}=\lambda^{2} x_{n+1}-\lambda a+a\left(-1+\lambda x_{n+1}-a-x_{n+1}\right) .
\end{gathered}
$$

Thus, we need to show that $x_{n+1}>-\frac{a+2}{1-\lambda}$ implies

$$
\lambda^{2} x_{n+1}-\lambda a+a\left(-1+\lambda x_{n+1}-a-x_{n+1}\right)>\frac{a}{1+\lambda},
$$


i.e.,

$$
\left(\lambda^{2}+a \lambda-a\right) x_{n+1}-a(\beta+1)>\frac{a}{1+\lambda} .
$$

Since $\lambda^{2}+a \lambda-a>1$, it suffies to show 4.5 for $x_{n+1}=-\frac{a+2}{1-\lambda}$, i.e.,

$$
\left(\lambda^{2}+a \lambda-a\right)\left(-\frac{a+2}{1-\lambda}\right)-a(\beta+1)>\frac{a}{1+\lambda} .
$$

But this is equivalent to

$$
\lambda^{2}(\beta+1)<0,
$$

which is true in the case we are considering. We see that the point $\left(x_{n+3},-1\right)$ belongs to the segment connecting the points $-A$ and $-B$, which is invariant for the map $f^{2}$ thanks to Lemma 4. Hence the trajectory converges to the 2-periodic orbit.

3 Next, we consider a trajectory which starts at a point $D^{\prime}$ on the line $s=1$ to the left of the point $A$ in the parallelogram $\Pi$. For this trajectory, further odd iterations $f^{k}\left(D^{\prime}\right)$ lie in the interior of $L$, while the even iterations $f^{k}\left(D^{\prime}\right)$ belong to the line $s=1$, and the $x$-coordinate of the even iterations monotonically increases until the trajectory reaches the segment $A B$. (This behaviour is similar to the behaviour that we considered in paragraph 2). Hence, such a trajectory also converges to the 2-periodic orbit.

Any trajectory that starts in the parallelogram $\Pi$, but not on the lines $s= \pm 1$ and not on the segment of equilibrium points, thanks to Lemma 3 will stay inside $\Pi$. It reaches one of the lines $s= \pm 1$ in several iterations due to the condition $\beta<-1$. Thus, we see that all the trajectories that start in the parallelogram $\Pi$ except for the segment of equilibrium points, converge to the 2-periodic orbit.

4 Finally, let us consider a trajectory that starts to the right of the parallelogram $\Pi$. Since $\beta<0$, this trajectory reaches the line $s=-1$ after one iteration. If it reaches this line to the right of the equilibrium point $F$, then it will move to the left along the line $s=-1$ and converge to the equilibrium point $F$ from the right. On the other hand, if a trajectory reaches the line $s=-1$ at a point, which lies to the left of the point $F$, then this point belongs to $\Pi$. This can be shown exactly in the same way as we did in Section 4.2.2. Therefore, such a trajectory converges to the 2-periodic orbit. We conclude that the 2-periodic orbit is stable and its basin of attraction contains the parallelogram $\Pi$ with the exception of equilibrium points. However, some trajectories from outside the parallelogram $\Pi$ are attracted to the semi-stable equilibrium points $E$ and $F$.

\subsection{Case $(d)$}

In this case, $a<0$ and the segment $E F$ has a negative slope (see Fig. 4.4). Like in Section 4.3, there exists a 2-periodic orbit $\pm Q$ defined by (2.4). Let $A$ be as in 4.4.

First, we note that if a point $\left(x_{n},-1\right)$ satisfies $x_{n} \leq-A_{x}$, then $x_{n+1}>\frac{a+2}{1-\lambda}, s_{n+1}=1$. Hence the half-line $\left\{(x, s): x \leq-A_{x}, s=-1\right\}$ is mapped to itself under $f^{2}$. Since $x_{n+2}=\lambda^{2} x_{n}-\lambda a+a$ and $\lambda^{2}<1$, any trajectory starting at this half-line converges to the 2-periodic orbit $Q$. 


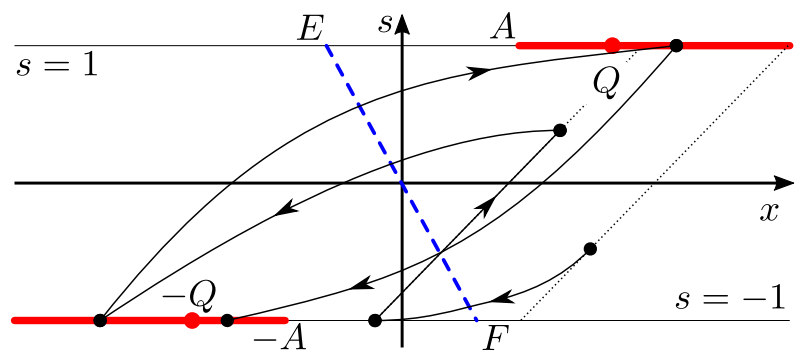

Figure 4.4: Case $\lambda<0, \beta<-1$. Red half-lines are mapped to themselves by $f^{2}$.

If a point belongs to the open segment $\left\{(x, s):-A_{x}<x<\frac{-a}{1-\lambda}, s=-1\right\}$, then its trajectory enters the half-line $\left\{(x, s): x \leq-A_{x}, s=-1\right\}$ after finitely many iterations because $\beta<-1$ (like in Section 4.3). Hence, the half-line $\left\{(x, s): x<\frac{-a}{1-\lambda}, s=-1\right\}$ belongs to the basin of attraction of the 2-periodic orbit. If a point belongs to the half-line $\left\{(x, s): x>\frac{-a}{1-\lambda}, s=-1\right\}$, then its first iteration is in the half-line $\left\{(x, s): x<\frac{-a}{1-\lambda}, s=\right.$ $-1\}$ because $\lambda<0$. Hence, we conclude that the lines $s=-1$ and $s=1$ (except for the equilibria $F$ and $E$, respectively) belong to the basin of attraction of the 2-periodic orbit.

Finally, all trajectories that start inside the strip $-1<s<1$, except for the equilibrium points, will reach one of the lines $s= \pm 1$ after finitely many iterations because $\beta<-1$. Therefore, the 2 -periodic orbit attracts all the trajectories except for the equilibrium points and their pre-images.

\subsection{Case $(b), \lambda<0$}

4.5.1 $-1<\beta<0$

For the point $\left(x_{n}, s_{n}\right)$ to the right of the parallelogram $\Pi$, one has

$$
x_{n}-s_{n}>p^{*}=1-x^{*}=1-\frac{a}{1-\lambda},
$$

and so $x_{n+1}<\lambda p^{*}-\beta=-x^{*}$, hence the point $\left(x_{n+1}, s_{n+1}\right)$ lies to the left of the equilibrium $F$ on the line $s=-1$, see Fig. 4.5(a). Due to 4.1), for the point $\left(x_{n}, s_{n}\right)$ to the left of $\Pi$, its image will lie on the line $s=1$ to the right of the point $E$.

Now, we prove that every trajectory enters П. Arguing by contradiction, let us show that if a trajectory never entered $\Pi$, then the distance from the trajectory to $\Pi$ would exponentially decrease. This would imply that such a trajectory converges to a 2-periodic orbit, because, as we have seen, its points belong to the union of the lines $s= \pm 1$ and the sign of $s_{n}$ alternates at every iteration. However, this is impossible as a 2-periodic orbit does not exist in the case we are considering.

In order to see that the distance from a trajectory to $\Pi$ exponentially decreases, it is sufficient to establish the inequality

$$
q\left(\frac{a}{1-\lambda}-2-x_{n}\right)>\lambda x_{n}-a+\frac{a}{1-\lambda}-2
$$

for $x_{n}<\frac{a}{1-\lambda}-2$ and some $q \in(-\lambda, 1)$ independent of $n$. This inequality can be written 
as

$$
x_{n}<\frac{a-\left(\frac{a}{1-\lambda}-2\right)(1-q)}{q+\lambda} .
$$

Thus we need to show that

$$
\frac{a}{1-\lambda}-2<\frac{a-\left(\frac{a}{1-\lambda}-2\right)(1-q)}{q+\lambda},
$$

which is equivalent to $(1+\lambda)\left(\frac{a}{1-\lambda}-2\right)<a$ and, further, to $\beta \lambda<1$. Since the last inequality is true in the case being considered, we can use any $q \in(-\lambda, 1)$. The above argument shows that every trajectory enters the parallelogram $\Pi$.

Since $\beta \in(-1,0)$ and $\lambda \in(-1,0)$, it follows from Lemma 3 that $\Pi$ is invariant for the map $f$. Further, we note that if some iteration of a point from $\Pi$ is mapped in the interior of $L$, then the trajectory converges to an equilibrium due to $|\beta|<1$, see Fig. 4.5(a).

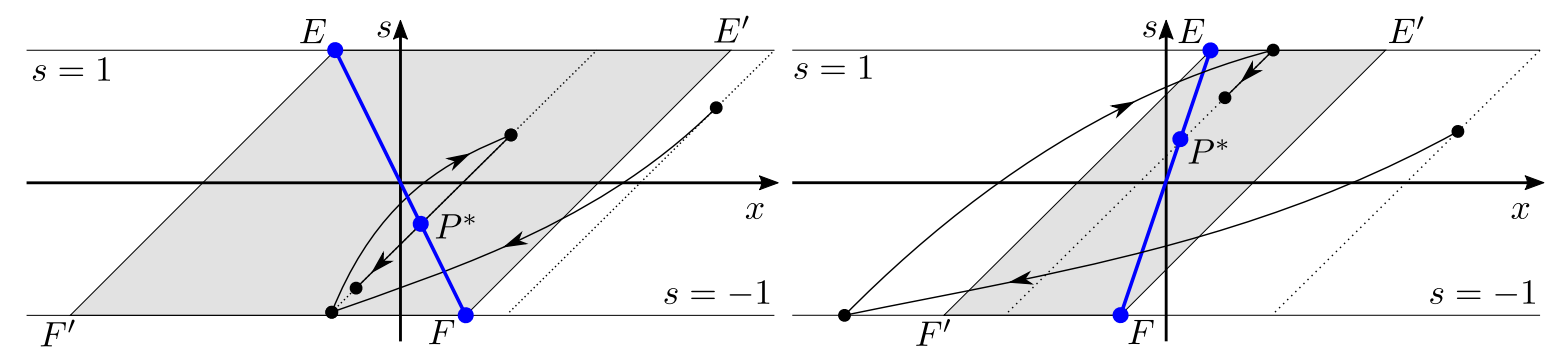

(a) $-1<\beta<0$.

(b) $0 \leq \beta<1$.

Figure 4.5: Theorem $1 \mid(b)$, Case $\lambda<0$.

Finally, let us show that a trajectory cannot jump from the line $s=1$ to the line $s=-1$ and back all the time. Indeed, if this was the case, then a point $\left(x_{n}, 1\right)$ from this trajectory would satisfy $x_{n}>1$ and the point $\left(x_{n+1},-1\right)$ would satisfy $x_{n+1}=\lambda x_{n}+a<$ -1 . But inequalities $-1<\beta$ and $x_{n}>1$ imply that $-1-\left(\lambda x_{n}+a\right)<x_{n}-1$. In other words, $0<-1-x_{n+1}<x_{n}-1$ and, similarly, $0<x_{n+2}-1<-1-x_{n+1}$. Therefore, this trajectory would converge to the 2 -periodic orbit, which does not exist in this case. This contradiction implies that every trajectory converges to an equilibrium point.

\subsection{2 $0 \leq \beta<1$}

In this case, $a>0$ and so the slope of the segment $E F$ is greater than 1 (see Fig. 4.5(b)). If a trajectory starts in $\Pi$, then it converges to an equilibrium point $P^{*} \in E F$ along the line $p=$ const.

A trajectory starting to the right of the parallelogram $\Pi$ moves along the line $p=$ const down and left until it reaches the line $s=-1$. At this point, or at the next iteration step, the trajectory reaches a point $\left(x_{n},-1\right)$ that lies to the left of the equilibrium point $F$ because $\lambda<0$. If $\left(x_{n},-1\right) \in \Pi$, then the trajectory converges to an equilibrium as we have seen above. If the point $\left(x_{n},-1\right)$ lies to the left of the parallelogram $\Pi$, then let us show that the absolute value $\left|x_{n}-s_{n}\right|=-1-x_{n}$ of the $p$-coordinate of this point is less than the absolute value $\left|x_{n-1}-s_{n-1}\right|=x_{n-1}-s_{n-1}$ of the $p$-coordinate of its preimage $\left(x_{n-1}, s_{n-1}\right)$. Since $x_{n}=\lambda x_{n-1}+a s_{n-1}$ and $s_{n}=-1$, we want to show that

$$
-\left(\lambda x_{n-1}+a s_{n-1}+1\right)<\left(x_{n-1}-s_{n-1}\right) q
$$


with some $q \in(-\lambda, 1)$ independent of the point $\left(x_{n-1}, s_{n-1}\right)$. Equivalently,

$$
x_{n-1}-s_{n-1}>\frac{-\beta s_{n-1}-1}{q+\lambda} .
$$

Indeed, since the point $\left(x_{n-1}, s_{n-1}\right)$ lies to the right of $\Pi$, we have $x_{n-1}-s_{n-1}>\frac{-a}{1-\lambda}+1$ and it remains to show that

$$
\frac{-a}{1-\lambda}+1>\frac{-\beta s_{n-1}-1}{q+\lambda}
$$

This inequality is equivalent to

$$
1+q>-\beta s_{n-1}(1-\lambda)+\beta(q+\lambda) .
$$

If we set $q=-\lambda+\varepsilon$ with a sufficiently small $\varepsilon>0$, then $q \in(0,1)$ and the inequalities $\left|s_{n-1}\right| \leq 1$ and $0 \leq \beta<1$ imply that

$$
-\beta s_{n-1}(1-\lambda)<1-\lambda, \quad \beta(q+\lambda)=\varepsilon(\lambda+a)<\varepsilon,
$$

hence the relation 4.7 holds.

Since $q$ in (4.6) does not depend on $\left(x_{n-1}, s_{n-1}\right)$ and the segment connecting the points $P_{1}=(1,1)$ and $P_{2}=(-1,-1)$ belongs to the interior of $\Pi$, all trajectories that start outside the parallelogram $\Pi$ will eventually enter $\Pi$ and converge to one of the equilibrium points.

\subsection{Case $(e)$}

In this case, $a>0$ and the slope of the segment $E F$ is positive and less than 1 (see Fig. 4.6.).

1 Denote by $l_{1}$ and $l_{2}$ the open half-lines starting at the point $E$ on the upper boundary of the strip $L$ :

$$
l_{1}=\left\{(x, s): x>\frac{a}{1-\lambda}, s=1\right\}, \quad l_{2}=\left\{(x, s): x<\frac{a}{1-\lambda}, s=1\right\}
$$

and by $l_{3}$ and $l_{4}$ the half-lines starting from the point $F$ on the lower boundary of the strip $L$ :

$$
l_{3}=\left\{(x, s): x<-\frac{a}{1-\lambda}, s=-1\right\}, \quad l_{4}=\left\{(x, s): x>-\frac{a}{1-\lambda}, s=-1\right\} .
$$

From the condition $\lambda<0$ it follows that $f\left(l_{2}\right) \subseteq l_{1}$ and $f\left(l_{4}\right) \subseteq l_{3}$. Also from Lemma 1 it follows that for any point $\left(x_{n}, s_{n}\right)$ such that $x_{n}>\frac{a s_{n}}{1-\lambda}$ one has $x_{n+1}<x_{n}$. Thus, starting from $l_{1}$, any trajectory arrives after finitely many iterations to the closed halfline $\overline{l_{3}}$. Hence, we can define the first-hitting map $\mathcal{P}: l_{1} \rightarrow \overline{l_{3}}$ as $\mathcal{P}(A)=f^{k}(A)$ where $f^{k}(A) \in \overline{l_{3}}$ and $f^{i}(A) \notin \overline{l_{3}}$ for $i=0, \ldots, k-1$. This map can be represented by the scalar function $T:\left(\frac{a}{1-\lambda}, \infty\right) \rightarrow\left[\frac{a}{1-\lambda}, \infty\right)$ defined by the formula

$$
(-T(x),-1)=\mathcal{P}(x, 1), \quad x \in\left(\frac{a}{1-\lambda}, \infty\right) .
$$

It is convenient to set $T\left(\frac{a}{1-\lambda}\right)=\frac{a}{1-\lambda}$ and consider $T$ as a map of the half-line $\left[\frac{a}{1-\lambda}, \infty\right)$ into itself. 
2 In this part, we describe the structure of the function $T(x)$. We begin with the following observation.

Lemma 5. Let $\left(x_{0}, 1\right) \in l_{1}$ be a point such that the first $n-1$ iterations of it under the map $f$ belong to the line $p=$ const. Then for any $m \leq n$ we have

$$
x_{m}=\beta^{m} x_{0}-a\left(x_{0}-1\right) \sum_{i=0}^{m-1} \beta^{i} .
$$

Proof. For $m=1$ equation 4.9 is obvious. Suppose that 4.9 holds for $m<n$. Then

$$
x_{m+1}=\lambda x_{m}+a\left(1+x_{m}-x_{0}\right)=\beta^{m+1} x_{0}-a\left(x_{0}-1\right) \sum_{i=0}^{m} \beta^{i} .
$$

Let us show that for any $k \in \mathbb{N}$ there exists a unique point $\left(r_{k}, 1\right) \in l_{1}$ such that its first $k-1$ iterations under the map $f$ belong to the line $p=$ const and its $k$-th iteration is $\left(r_{k}-2,-1\right)$. Setting $m=k, x_{0}=r_{k}$, and $x_{m}=r_{k}-2$ in 4.9 ), we obtain

$$
r_{k}=\frac{2+a \frac{1-\beta^{k}}{1-\beta}}{1-\beta^{k}+a \frac{1-\beta^{k}}{1-\beta}}
$$

(it is easy to see that the denominator does not vanish as long as $f^{i}\left(r_{k}, 1\right)$ belongs to the interior of $L$ for $i=1, \ldots, k-1)$.

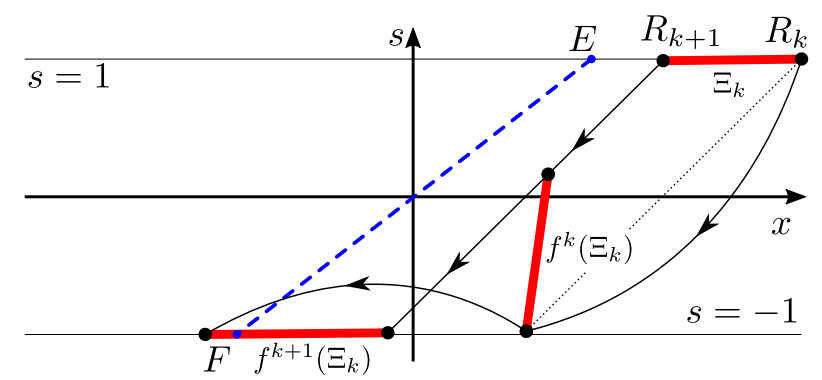

Figure 4.6: The segment $\Xi_{k}$ and its images $f^{k}\left(\Xi_{k}\right)$ and $f^{k+1}\left(\Xi_{k}\right)$.

Next we show that for any $k \in \mathbb{N}$ there exists a unique point $\left(q_{k}, 1\right) \in l_{1}$ such that its first $k-1$ iterations under the map $f$ belong to the line $p=$ const and its $k$-th iteration is $F$.

Obviously, $q_{1}=\frac{1}{\lambda}\left(-a-\frac{a}{1-\lambda}\right)$. Set $R_{i}=\left(r_{i}, 1\right)$ and consider the $k$-th iteration of the segment $\Xi_{k}=R_{k+1} R_{k}$. The point $R_{k}$ is mapped to the point $\left(r_{k}-2,-1\right)$, and the image of the point $R_{k+1}$ belongs to the interior of $L$. Hence, $f^{k}\left(\Xi_{k}\right)$ is a segment which lies entirely to the right of the segment $E F$ and all its points except $f^{k}\left(R_{k}\right)$ belong to the interior of $L$. Consider the $(k+1)$-st iteration of $\Xi_{k}$. The point $f^{k+1}\left(R_{k}\right)$ lies on the line $s=-1$ to the left of the point $F$, while $f^{k+1}\left(R_{k+1}\right)=\left(r_{k+1}-2,-1\right)$. Hence, $f^{k+1}\left(\Xi_{k}\right)$ is a segment on the line $s=-1$ and $F \in f^{k+1}\left(\Xi_{k}\right)$, see Fig. 4.6. Hence, there exists a point 
$q_{k+1} \in\left(r_{k+1}, r_{k}\right)$ for each $k \in \mathbb{N}$. Now $q_{k}$ can be found in a unique way using Lemma 5 by setting $m=k, x_{0}=q_{k}$, and $x_{m}=\frac{-a}{1-\lambda}$ :

$$
q_{k}=\frac{-\frac{a}{1-\lambda}-a \frac{1-\beta^{k}}{1-\beta}}{\beta^{k}-a \frac{1-\beta^{k}}{1-\beta}} .
$$

Since $a>0$ and $\beta>1$, the denominator does not vanish. Note that

$$
q_{1}>r_{1}>q_{2}>r_{2}>\cdots>\frac{a}{1-\lambda}, \quad q_{k}, r_{k} \rightarrow \frac{a}{1-\lambda} \text { as } k \rightarrow \infty .
$$

It follows from the relation $\left(r_{k}-2,-1\right)=f^{k}\left(R_{k}\right)$ that $f^{k+1}\left(R_{k}\right)=\left(\lambda\left(r_{k}-2\right)-a,-1\right)$, i.e.,

$$
T\left(r_{k}\right)=a-\lambda\left(r_{k}-2\right) .
$$

Combining (4.12) and (4.13), we see that

$$
T\left(r_{1}\right)>T\left(r_{2}\right)>\ldots, \quad T\left(r_{k}\right)-\frac{a}{1-\lambda} \rightarrow T_{*}>0,
$$

where

$$
T_{*}=\frac{2 \lambda(1-a-\lambda)}{1-\lambda} .
$$

Furthermore, the above argument shows that the function $T(x)$ is continuous and piecewise linear for $x \in(a /(1-\lambda), \infty)$. Using (4.10), 4.11), and 4.13), we see that it increases on the intervals $\left(q_{1}, \infty\right)$ and $\left(q_{k}, r_{k-1}\right), k=2,3, \ldots$ with

$$
T^{\prime}(x)=-\lambda, x \in\left(q_{1}, \infty\right), \quad T^{\prime}(x)=-\left(\beta^{k}-a \frac{1-\beta^{k}}{1-\beta}\right), x \in\left(q_{k}, r_{k-1}\right),
$$

respectively, and decreases on the intervals $\left(r_{k}, q_{k}\right), k \in \mathbb{N}$, with

$$
T^{\prime}(x)=-\lambda\left(\beta^{k}-a \frac{1-\beta^{k}}{1-\beta}\right), \quad x \in\left(r_{k}, q_{k}\right),
$$

see Fig. 4.7. Every point $x \in\left[q_{1}, \infty\right)$ possesses the property that $f(x, 1) \in l_{3}$. Every point $x \in\left[q_{k+1}, q_{k}\right), k \in \mathbb{N}$, possesses the property that the $(k+1)$ th iteration of the point $(x, 1) \in l_{1}$ under the map $f$ reaches the half-line $l_{3}$ for the first time.

3 Now we show that system (2.2) has periodic orbits of all sufficiently large periods. Fix $k_{1} \in \mathbb{N}$ such that

$$
q_{k_{1}}-\frac{a}{1-\lambda}<T_{*}
$$

where $T_{*}>0$ is given by 4.15$)$. Note that $T\left(\left[r_{k_{1}}, q_{k_{1}}\right]\right)=\left[\frac{a}{1-\lambda}, T\left(r_{k_{1}}\right)\right]$. Fix $k_{2} \in \mathbb{N}$ such that

$$
q_{k_{2}}<T\left(r_{k_{1}}\right)
$$

For any $m \geq k_{2}$, denote by $\Theta_{m}$ the subsegment of $\left[r_{k_{1}}, q_{k_{1}}\right]$ such that $T\left(\Theta_{m}\right)=\left[r_{m}, q_{m}\right]$. It follows from (4.14) and 4.18) that

$$
T^{2}\left(\Theta_{m}\right) \supset\left[\frac{a}{1-\lambda}, \frac{a}{1-\lambda}+T_{*}\right] \supset \Theta_{m} .
$$

Hence, the map $T^{2}$ has a fixed point in $\Theta_{m}$. Due to the argument in part 2 of this section, the corresponding periodic solution of the system (2.2) will be of period $k_{1}+m+2$. Hence, for any $k \geq k_{1}+k_{2}+2$, system (2.2) has $k$-periodic orbit. 


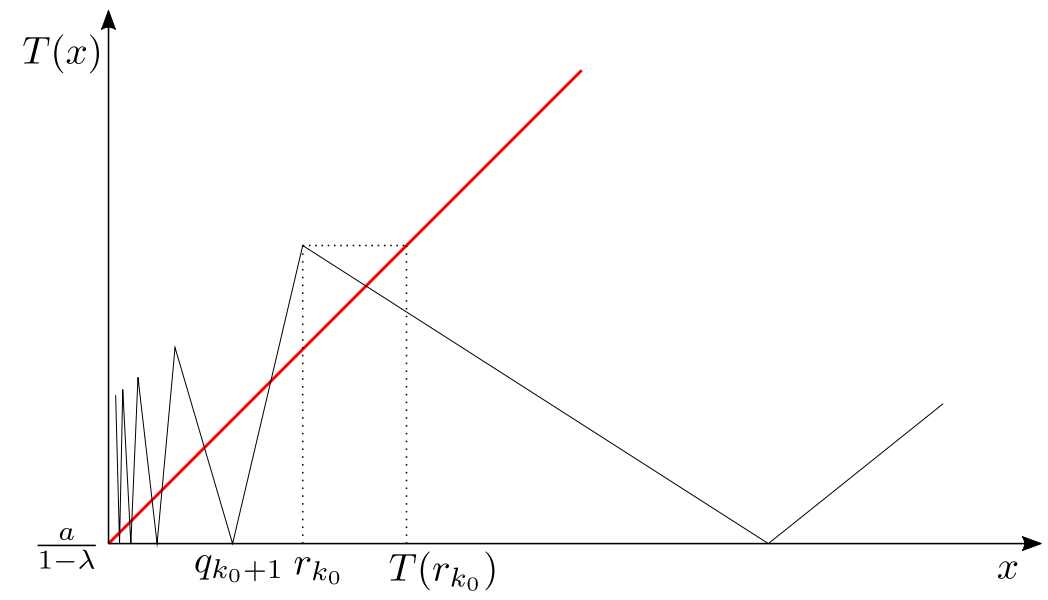

Figure 4.7: Graph of the map $T(x)$ for $x \in\left(\frac{a}{1-\lambda}, \infty\right)$.

4 To complete the proof of statement (e), it remains to show that system (2.2) has no more than one stable periodic orbit. In this part, we find a necessary and sufficient condition for the map $T(x)$ to have fixed points in the interval $\left(q_{k+1}, q_{k}\right)$ (obviously, $T(x)$ has no fixed points for $x \geq q_{1}$ because $T^{\prime}(x)=-\lambda \in(0,1)$ for all $\left.x>q_{1}\right)$. Then we show that at most one fixed point of $T(x)$ can be stable. Finally, in part 5 , we prove that all the periodic orbits of $T(x)$ with minimal period greater than 1 are unstable.

Lemma 6. The map $T(x)$ has a fixed point in the interval $\left(q_{k+1}, q_{k}\right)$ if and only if

$$
1+\lambda \beta^{k} \leq 0 .
$$

The period of the corresponding orbit of system 2.2 equals $(2 k+2)$.

Proof. The interval $\left(q_{k+1}, q_{k}\right)$ contains a fixed point if and only if

$$
T\left(r_{k}\right) \geq r_{k}
$$

see Fig. 4.7. Using formulas 4.10 and $(4.13)$, we see that 4.20 is equivalent to

$$
\frac{a+2 \lambda}{1+\lambda} \geq \frac{2+a \frac{1-\beta^{k}}{1-\beta}}{1-\beta^{k}+a \frac{1-\beta^{k}}{1-\beta}},
$$

which can be rewritten as 4.19).

Note that, given $a$ and $\lambda$, inequality (4.19) holds for all sufficiently large $k$. We denote by $k_{0}=k_{0}(\lambda, a)$ the smallest $k$ with this property.

Now we fix $a$ and $\lambda$ and an arbitrary $k \geq k_{0}(\lambda, a)$ and study the stability of the fixed points of $T(x)$ in the interval $\left(q_{k+1}, q_{k}\right)$. First, note that if (4.19) holds as an equality, then the interval $\left(q_{k+1}, q_{k}\right)$ contains a unique fixed point $r_{k}$. It is unstable because the slope of the graph of $T(x), x \in\left(q_{k+1}, r_{k}\right)$ is positive and greater than one. Assume that (4.19) holds as a strict inequality, i.e,

$$
1+\lambda \beta^{k}<0 .
$$


Then there are two fixed points on the interval $\left(q_{k+1}, q_{k}\right)$. The left one belongs to the interval $x \in\left(q_{k+1}, r_{k}\right)$ and is unstable (as in the previous case). The right one belongs to the interval $\left(r_{k}, q_{k}\right)$. It is stable if and only if

$$
T^{\prime}(x) \in[-1,0), \quad x \in\left(r_{k}, q_{k}\right) .
$$

Combining (4.22) and (4.17), we conclude that the fixed point from the interval $\left(r_{k}, q_{k}\right)$ is stable if and only if

$$
\lambda \beta^{k}+a-1 \geq 0
$$

Lemma 7. Inequalities (4.21) and (4.23) are either incompatible for all $k \geq k_{0}$, or they hold for $k=k_{0}$ only.

Proof. In the parameter plane $(\lambda, \beta)$, we introduce the regions $\Omega_{k}$ (see $\left.(2.5)\right)$. Assume that for given $(\lambda, \beta)$, inequalities (4.21) and (4.23) hold for $k_{1}$ and $k_{2}$. Then, $(\lambda, \beta) \in \Omega_{k_{1}} \cap \Omega_{k_{2}}$. But $\Omega_{k_{1}}$ and $\Omega_{k_{2}}$ do not intersect for $k_{1} \neq k_{2}$. Hence, there exists no more than one $k \geq k_{0}$ for which inequalities (4.21) and (4.23) hold simultaneously. Assume that both inequalities hold for some $k>k_{0}$. Then inequality (4.21) holds also for $k_{0}$ by definition of $k_{0}$, while inequality 4.23 holds for $k_{0}$ due to the monotonicity of its left-hand side with respect to $k$. However, as we have just seen, both inequalities (4.21) and (4.23) cannot hold for two different values $k$ and $k_{0}$ simultaneously.

5 We denote by $r_{*}$ the (unique) fixed point of the map $T(x)$ in the interval $\left[r_{k_{0}}, q_{k_{0}}\right)$, where $k_{0}=k_{0}(\lambda, a)$ was introduced in part 4 . Recall that the map $T(x)$ has no fixed points for $x>r_{*}$. In particular, this implies that

$$
T(x)<x \text { for all } x>r_{*} .
$$

It remains to show that all the fixed points of any iteration of $T(x)$, except for the possibly stable fixed point $r_{*}$, are unstable.

First, we note that the fixed points of any iteration belong to the segment $\left[\frac{a}{1-\lambda}, T\left(r_{k_{0}}\right)\right]$. Indeed, if $x>T\left(r_{k_{0}}\right)$, then $x>r_{*}$, and (4.24) implies that either $T^{j}(x) \in\left[\frac{a}{1-\lambda}, T\left(r_{k_{0}}\right)\right]$ for some $j$, or $T^{j}(x) \rightarrow T\left(r_{k_{0}}\right)$ as $j \rightarrow \infty$.

Second, let us show that the segment $\left[\frac{a}{1-\lambda}, T\left(r_{k_{0}}\right)\right]$ is mapped onto itself under $T(x)$. Indeed, $\left[q_{k_{0}+1}, r_{k_{0}}\right] \subset\left[\frac{a}{1-\lambda}, T\left(r_{k_{0}}\right)\right]$ and $T\left(\left[q_{k_{0}+1}, r_{k_{0}}\right]\right)=\left[\frac{a}{1-\lambda}, T\left(r_{k_{0}}\right)\right]$. Hence, $\left[\frac{a}{1-\lambda}, T\left(r_{k_{0}}\right)\right] \subset$ $T\left(\left[\frac{a}{1-\lambda}, T\left(r_{k_{0}}\right)\right]\right)$. On the other hand, $T(x) \leq T\left(r_{k_{0}}\right)$ for $x \leq r_{*}$ due to the monotonicity of $T\left(r_{k}\right)$ (see (4.14)) and $T(x)<x \leq T\left(r_{k_{0}}\right)$ for $x \in\left[r_{*}, T\left(r_{k_{0}}\right)\right]$ due to (4.24). Thus,

$$
T\left(\left[\frac{a}{1-\lambda}, T\left(r_{k_{0}}\right)\right]\right)=\left[\frac{a}{1-\lambda}, T\left(r_{k_{0}}\right)\right]
$$

and it suffices to study the iterations of $T(x)$ on the segment $\left[\frac{a}{1-\lambda}, T\left(r_{k_{0}}\right)\right]$. We consider two cases: fixed point $r_{*}$ being stable or unstable.

5.1 Assume that the fixed point $r_{*}$ is stable. Let us show that

$$
T\left(r_{k_{0}}\right) \in\left[r_{*}, q_{k_{0}}\right) .
$$


Obviously $T\left(r_{k_{0}}\right) \geq r_{*}$. On the other hand, since

$$
\left|T^{\prime}(x)\right| \leq 1 \quad \text { for all } x \in\left(r_{k_{0}}, q_{k_{0}}\right)
$$

it follows that

$$
q_{k_{0}}-r_{k_{0}} \geq T\left(r_{k_{0}}\right)-\frac{a}{1-\lambda}>T\left(r_{k_{0}}\right)-r_{k_{0}}
$$

i.e., $T\left(r_{k_{0}}\right) \leq q_{k_{0}}$.

Next, we show that the segment $\left[r_{k_{0}}, T\left(r_{k_{0}}\right)\right]$ is invariant under $T$, i.e.,

$$
T\left(\left[r_{k_{0}}, T\left(r_{k_{0}}\right)\right]\right) \subset\left[r_{k_{0}}, T\left(r_{k_{0}}\right)\right] .
$$

Since $T(x)$ is linear on this segment, we need to check the images $T\left(r_{k_{0}}\right)$ and $T^{2}\left(r_{k_{0}}\right)$ of the end points only. Obviously, $T\left(r_{k_{0}}\right)$ belongs to this segment. Moreover, relations (4.25) and (4.26) show that all the iterations of $r_{k_{0}}$ under the map $T$ belong to the segment $\left[r_{k_{0}}, T\left(r_{k_{0}}\right)\right]$ (and converge to $r_{*}$ ). In particular, $T^{2}\left(r_{k_{0}}\right)$ belongs to this interval.

Now we are ready to prove that the fixed points of any iteration of $T(x)$, except for $r_{*}$, are unstable. Assume, to the contrary, that $x_{*} \in\left(\frac{a}{1-\lambda}, T\left(r_{k_{0}}\right)\right]$ is a stable fixed point of $T^{j_{*}}(x)$ for some $j_{*} \geq 2$ and $x_{*} \neq r_{*}$. We have seen in part 4 of this section that $\left|T^{\prime}(x)\right|>1$ for all $x \in\left(r_{k+1}, q_{k+1}\right) \cup\left(q_{k+1}, r_{k}\right), k \geq k_{0}$. Therefore, the only possibility for $x_{*}$ to be stable is that $T^{j}\left(x_{*}\right) \in\left[r_{k_{0}}, T\left(r_{k_{0}}\right)\right]$ for some $j \in \mathbb{N}$. However, all the trajectories entering this segment converge to $r_{*}$ due to 4.26) and (4.27).

5.2 Assume that the fixed point $r_{*}$ is unstable. Then $k_{0}>1$ (otherwise, $\left|T^{\prime}\left(r_{*}\right)\right|=$ $\left.\lambda^{2}<1\right)$. It follows from the monotonicity of $T\left(r_{k}\right)$ (see (4.14) ) and (4.24) that $T\left(r_{k_{0}}\right)<$ $T\left(r_{k_{0}-1}\right)<r_{k_{0}-1}$, i.e.,

$$
\left[\frac{a}{1-\lambda}, T\left(r_{k_{0}}\right)\right] \subset\left[\frac{a}{1-\lambda}, r_{k_{0}-1}\right]
$$

But $\left|T^{\prime}(x)\right|>1$ for all $x \in\left(q_{k+1}, r_{k}\right) \cup\left(r_{k}, q_{k}\right), k \geq k_{0}$, due to part 4 of this section, and $\left|T^{\prime}(x)\right|=\left|\lambda^{-1} T^{\prime}\left(r_{*}\right)\right|>1$ for all $x \in\left(q_{k_{0}}, r_{k_{0}-1}\right)$ due to (4.16) and (4.17). This and (4.28) imply that the absolute values of all the slopes of the graph of $T(x)$ on the interval $\left[\frac{a}{1-\lambda}, T\left(r_{k_{0}}\right)\right]$ are greater than 1 . Hence, the same is true for any iteration of $T(x)$ on this interval. Therefore, all the fixed points of any iteration of $T$ are unstable. This completes the proof of statement $(e)$ of Theorem 1 .

\section{Proof of Theorem 2}

If $(\lambda, \beta) \in \Omega_{k}$ for some $k \in \mathbb{N}$, then, according to Lemmas 6 and 7 , the map $T$ has a stable fixed point in the interval $\left[r_{k}, q_{k}\right)$ and $k=k_{0}(\lambda, a)$. It corresponds to the stable $(2 k+2)$-periodic orbit of system (2.2). According to part 5.1 of this section, all the other periodic orbits are unstable.

If $(\lambda, a) \notin \bigcup_{k \in \mathbb{N}} \Omega_{k}$, then, according to Lemma 7, the map $T$ has no stable fixed points. Therefore, due to part 5.2 of this section, all the periodic orbits are unstable. 


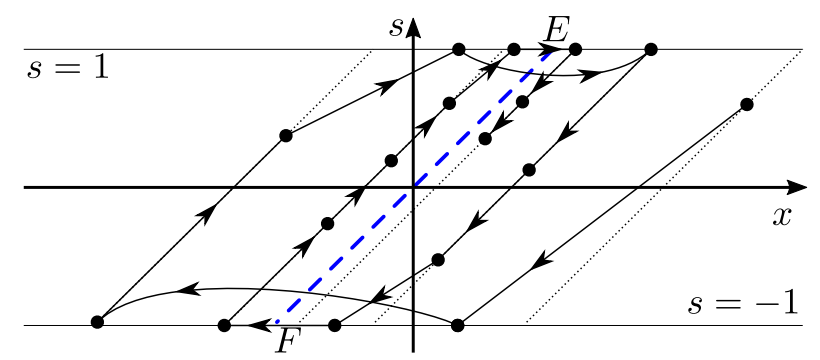

Figure 4.8: Case $\beta=1, \lambda<0$. The parallelogram $\Pi$ degenerates to the segment $E F$ with slope 1.

\subsection{Case $(f)$}

In this case, the parallelogram $\Pi$ degenerates into the segment of the equilibrium points EF with the slope 1 (see Fig. 4.8).

Let us consider a point $\left(x_{n}, s_{n}\right) \notin E F$. To be definite, assume that $p_{n}=x_{n}-s_{n}>0$. Denote by $\left(x_{n+k}, s_{n+k}\right)$ the first iteration that reaches the line $s=-1$ after the moment $n$, i.e., $s_{n+i}>-1$ for $i=0, \ldots, k-1$ and $s_{n+k}=-1$ (if $s_{n}=-1$ we agree that $k=0$ ). If $p_{n+k}=0$, then the trajectory ends at the point $F$. If $p_{n+k}>0$, then $0<p_{n+k} \leq p_{n+k-1}=\cdots=p_{n}$ and

$$
p_{n+k+1}=\lambda x_{n+k}+a s_{n+k}-s_{n+k+1}=\lambda x_{n+k}-a+1=\lambda x_{n+k}+\lambda=\lambda p_{n+k},
$$

where we use $a=1-\lambda$. If $p_{n+k}<0$, then $p_{n+k}<0<p_{n+k-1}=\cdots=p_{n}$ and

$$
p_{n+k}=\lambda x_{n+k-1}+a s_{n+k-1}-s_{n+k}=\lambda p_{n+k-1}+s_{n+k-1}-s_{n+k} \geq \lambda p_{n+k-1},
$$

hence

$$
\left|p_{n+k}\right| \leq|\lambda|\left|p_{n+k-1}\right| \text {. }
$$

Inequalities (4.29) and 4.30 and similar inequalities that hold for ascending parts of trajectories, due to (4.1), show that the trajectory either ends up at $E$ or $F$, or converges to the segment $E F$.

\subsection{Case $(g)$}

For $\beta=-1$ it is straightforward to see that the parallelogram $\Sigma$, which is contained in $\Pi$, consists of 2-periodic orbits and the segment $E F$ of equilibrium points.

If $\lambda \geq 0$ (see Fig. 4.9(a)), then from Lemma 3 it follows that the parallelogram $\Pi$ is invariant under the map $f$ and if $\left(x_{n}, s_{n}\right) \in \Pi \backslash \Sigma$, then either $\left(x_{n+1}, s_{n+1}\right) \in \Sigma$ or $x_{n+1}<-1, s_{n+1}=-1$ or $x_{n+1}>1, s_{n+1}=1$. But, since $\lambda \geq 0$, relations $x_{n+1}<-1$, $s_{n+1}=-1$ and $f(-1,-1)=(1,1)$ imply $x_{n+2}<1, s_{n+2}=1$ and, similarly, relations $x_{n+1}>1, s_{n+1}=1$ and $f(1,1)=(-1,-1)$ imply $x_{n+2}>-1, s_{n+2}=-1$. In both cases, $\left(x_{n+2}, s_{n+2}\right) \in \Sigma$. Thus, $f^{2}$ maps $\Pi$ into $\Sigma$. On the other hand, the argument presented in Section 4.3 shows that a trajectory starting outside $\Pi$ either converges to the point $F$ along the line $s=-1$ from the right or to the point $E$ along the line $s=1$ from the left or meets the boundary of the strip $L$ inside $\Pi$. 


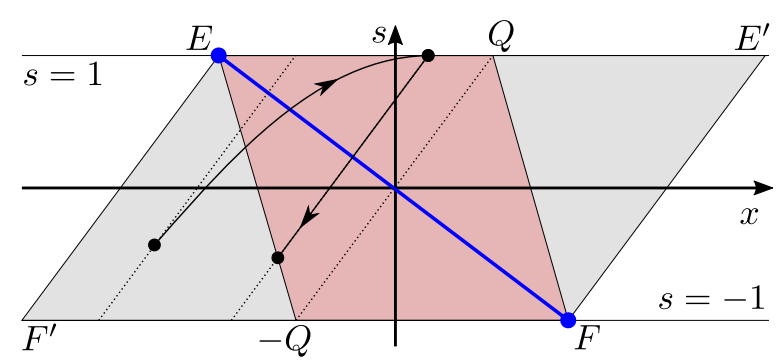

(a) $\lambda \geq 0$.

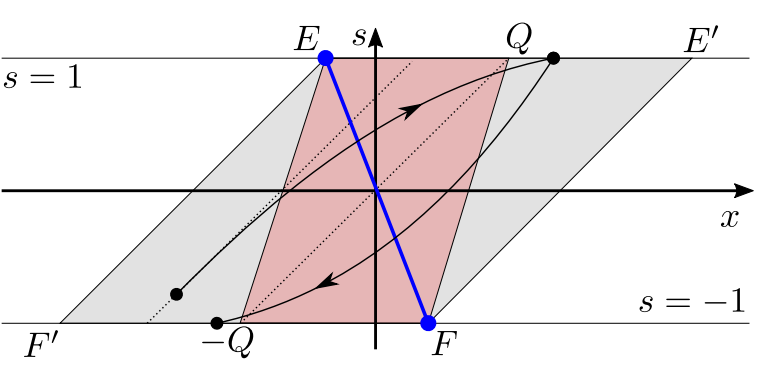

(b) $\lambda<0$

Figure 4.9: Parallelograms $\Sigma$ and $\Pi$ for $\beta=-1$.

If $\lambda<0$ (see Fig. 4.9(b)), then the argument used in Section 4.4 shows that the set $M=\{(x, s): x \leq-1, s=-1\} \cup\{(x, s): x \geq 1, s=1\}$ is invariant under the map $f$ and all the trajectories starting in $M$ converge to the 2-periodic orbit $(-1,-1),(1,1)$. If $\left(x_{n}, s_{n}\right) \in \Pi \backslash \Sigma$, then $\left(x_{n+1}, s_{n+1}\right) \in M \cup \Sigma$ because $\beta=-1$. Finally, if $\left(x_{n}, s_{n}\right) \notin \Pi$, then $\left(x_{n+k}, s_{n+k}\right) \in M \cup \Sigma$ for some $k \in \mathbb{N}$. This completes the proof of Theorem 1 .

\section{Acknowledgements}

NB acknowledges Saint-Petersburg State University (research grant 6.38.223.2014), the Russian Foundation for Basic Research (project No 16 - $01-00452$ ) and DFG project SFB 910. The work of PG was supported by the DFG Heisenberg Programme and DFG project SFB 910. DR was supported by NSF grant DMS-1413223.

\section{References}

[1] B. Appelbe, D. Flynn, H. Mcnamara, P. O'Kane, A. Pimenov, A. Pokrovskii, D. Rachinskii, and A. Zhezherun. Rate-independent hysteresis in terrestrial hydrology. Control Systems, IEEE, 29(1):44-69, 2009.

[2] B. Appelbe, D. Rachinskii, and A. Zhezherun. Hopf bifurcation in a Van der Pol type oscillator with magnetic hysteresis. Physica B: Condensed Matter, 403(2):301-304, 2008.

[3] K. J. Åström. Oscillations in systems with relay feedback. In Adaptive control, filtering, and signal processing (Minneapolis, MN, 1993), volume 74 of IMA Vol. Math. Appl., pages 1-25. Springer, New York, 1995.

[4] Z. Balanov, W. Krawcewicz, D. Rachinskii, and A. Zhezherun. Hopf bifurcation in symmetric networks of coupled oscillators with hysteresis. J. Dynam. Differential Equations, 24(4):713-759, 2012.

[5] L.M. Ball. Hysteresis in unemployment: Old and new evidence. Technical report, National Bureau of Economic Research, 2009.

[6] J. Barrdear. Towards a new Keynesian theory of the price level. Technical report, Bank of England and the Centre for Macroeconomics, 2015. 
[7] A. Belke, M. Göcke, and M. Günther. Exchange rate bands of inaction and playhysteresis in German exportssectoral evidence for some OECD destinations. Metroeconomica, 64(1):152-179, 2013.

[8] J. Bellaïche. On the path-dependence of economic growth. J. Math. Econom., 46(2):163-178, 2010.

[9] M. Brokate, S. McCarthy, A. Pimenov, A. Pokrovskii, and D. Rachinskii. Energy dissipation in hydrological systems due to hysteresis. Environmental Modeling \&6 Assessment, 16(4):313-333, 2011.

[10] M. Brokate, A. Pokrovskii, D. Rachinskii, and O. Rasskazov. Differential equations with hysteresis via a canonical example. In G. Bertotti and I.D. Mayergoyz, editors, The Science of Hysteresis, pages 127-291. Academic Press, Oxford, 2006.

[11] M. Brokate and J. Sprekels. Hysteresis and Phase Transitions. Springer-Verlag, New York, 1996.

[12] L. Christiano, M. Trabandt, and K. Walentin. DSGE models for monetary policy analysis. In Handbook of Monetary Economics, pages 285-367. Elsevier, 2011.

[13] R. Cross, M. Grinfeld, H. Lamba, and T. Seaman. Stylized facts from a thresholdbased heterogeneous agent model. Eur. Phys. J. B, 57(2):213-218, 2007.

[14] R. Cross, H. Hutchinson, H. Lamba, and D. Strachan. Reflections on Soros: Mach, Quine, Arthur and far-from-equilibrium dynamics. Journal of Economic Methodology, 20(4):357-367, 2013.

[15] R. Cross, H. McNamara, A. Pokrovskii, and D. Rachinskii. A new paradigm for modelling hysteresis in macroeconomic flows. Physica B: Condensed Matter, 403(2):231236, 2008.

[16] J. Darby, R. Cross, and L. Piscitelli. Hysteresis and unemployment: A preliminary investigation. In G. Bertotti and I.D. Mayergoyz, editors, The Science of Hysteresis, pages 667 - 699. Academic Press, Oxford, 2006.

[17] D. Davino, P. Krejčí, and C. Visone. Fully coupled modeling of magneto-mechanical hysteresis through thermodynamic compatibility. Smart Materials and Structures, 22(9):095009, 2013.

[18] P. De Grauwe. Booms and busts in economic activity: A behavioral explanation. Journal of Economic Behavior $\& 3$ Organization, 83(3):484-501, 2012.

[19] J.C. Driscoll and S. Holden. Behavioral economics and macroeconomic models. Journal of Macroeconomics, 41:133-147, 2014.

[20] M. Eleuteri, J. Kopfova, and P. Krejčí. A new phase field model for material fatigue in an oscillating elastoplastic beam. Discrete Contin. Dyn. Syst., 35(6):2465-2495, 2015. 
[21] G. Friedman, S. McCarthy, and D. Rachinskii. Hysteresis can grant fitness in stochastically varying environment. PloS ONE, 9(7):e103241, 2014.

[22] M. Göcke. Various concepts of hysteresis applied in economics. Journal of Economic Surveys, 16(2):167-188, 2002.

[23] M. Göcke and L. Werner. Play hysteresis in supply or in demand as part of a market model. Metroeconomica, 66(2):339-374, 2015.

[24] R. S. Harris. Pressure-volume curves of the respiratory system. Respiratory care, 50(1):78-99, 2005.

[25] R.V. Iyer and X. Tan. Control of hysteretic systems through inverse compensation. IEEE Control Syst. Mag., 29(1):83-99, 2009.

[26] M. Al Janaideh, S. Rakheja, and C.-Y. Su. A generalized Prandtl-Ishlinskii model for characterizing the hysteresis and saturation nonlinearities of smart actuators. Smart Materials and Structures, 18(4):045001, 2009.

[27] B. Jayawardhana, R. Ouyang, and V. Andrieu. Stability of systems with the Duhem hysteresis operator: the dissipativity approach. Automatica J. IFAC, 48(10):2657$2662,2012$.

[28] B.D. Keen. Output, inflation, and interest rates in an estimated optimizing model of monetary policy. Review of Economic Dynamics, 12(2):327-343, 2009.

[29] A. Krasnosel'skii and D. Rachinskii. Bifurcation of forced periodic oscillations for equations with Preisach hysteresis. Journal of Physics: Conference Series, 22(1):93$102,2005$.

[30] M.A. Krasnosel'skii and A.V. Pokrovskii. Systems with Hysteresis. Springer-Verlag, Berlin, 1989.

[31] P. Krejčí. Hysteresis, Convexity and Dissipation in Hyperbolic Equations. Gakkōtosho,Tokyo, 1996.

[32] P. Krejčí, H. Lamba, S. Melnik, and D. Rachinskii. Analytical solution for a class of network dynamics with mechanical and financial applications. Physical Review E, 90(3):032822, 2014.

[33] P. Krejčí, J.P. O'Kane, A. Pokrovskii, and D. Rachinskii. Stability results for a soil model with singular hysteretic hydrology. Journal of Physics: Conference Series, 268(1):012016, 2011.

[34] P. Krejčí and J. Sprekels. Elastic-ideally plastic beams and Prandtl-Ishlinskii hysteresis operators. Math. Methods Appl. Sci., 30(18):2371-2393, 2007.

[35] M. Kunze and M.D.P. Monteiro Marques. An introduction to Moreau's sweeping process. In Impacts in mechanical systems (Grenoble, 1999), volume 551 of Lecture Notes in Phys., pages 1-60. Springer, Berlin, 2000. 
[36] H. Lamba. Implausible equilibrium solutions in economics and finance. SSRN, 2408013, 2014.

[37] H. Lamba and T. Seaman. Market statistics of a psychology-based heterogeneous agent model. International Journal of Theoretical and Applied Finance, 11(07):717$737,2008$.

[38] N.G. Mankiw and R. Reis. Sticky information in general equilibrium. Journal of the European Economic Association, 5(2-3):603-613, 2007.

[39] I.D. Mayergoyz. Mathematical Models of Hysteresis and Their Applications. Electromagnetism. Elsevier Science, New York, 2003.

[40] J.-J. Moreau. On unilateral constraints, friction and plasticity. In New variational techniques in mathematical physics (Centro Internaz. Mat. Estivo (C.I.M.E.), II Ciclo, Bressanone, 1973), pages 171-322. Edizioni Cremonese, Rome, 1974.

[41] J.-Y. Parlange, R. Haverkamp, G. Sander, W.L. Hogarth, and R.D. Braddock. Comments on "General scaling rules of the hysteretic water retention function based on Mualem's domain theory" by Y. Mualem \& A. Beriozkin. European Journal of Soil Science, 61(6):1113-1114, 2010.

[42] L. Prandtl. Ein Gedankenmodell zur kinetischen Theorie der festen Körper. ZAMMJournal of Applied Mathematics and Mechanics/Zeitschrift für Angewandte Mathematik und Mechanik, 8(2):85-106, 1928.

[43] K. Rao, A.M. Sbordone, A. Tambalotti, and K.J. Walsh. Policy analysis using DSGE models: an introduction. Economic Policy Review, 16(2), 2010.

[44] M. Ruderman and T. Bertram. Modified Maxwell-slip model of presliding friction. In Proc. 18th IFAC World Congress, pages 10764-10769, 2011.

[45] I. Rychlik. A new definition of the rainflow cycle counting method. International journal of fatigue, 9(2):119-121, 1987.

[46] J.B. Taylor. The inflation/output variability trade-off revisited. In Goals, Guidelines and Constraints Facing Monetary Policymakers, volume 38 of Conference Series, pages 21-38. Federal Reserve Bank of Boston, 1994.

[47] A. Visintin. Differential Models of Hysteresis. Applied Mathematical Sciences. Springer, Berlin, 1994. 